N. Mochizuki

Nagoya Math. J.

Vol. 52 (1973), 53-59

\title{
HOLOMORPHIC EXTENSION OF CONTINUOUS, WEAKLY HOLOMORPHIC FUNCTIONS ON GERTAIN ANALYTIC VARIETIES
}

\author{
NOZOMU MOCHIZUKI
}

\section{§ 1. Introduction}

Let $M, N$ be connected complex submanifolds of a neighborhood of the origin $0 \in C^{d}$, the space of $d$ complex variables, such that $0 \in M \cap N$. We shall suppose throughout that $M \not \subset N$ and $N \not \subset M$ in any neighborhood of 0 . Let $X=M \cup N . \quad X$ is an analytic subvariety with the irreducible branches $M$ and $N$. Let $\Delta$ be a neighborhood of 0 in $C^{d}$. We consider the following proposition:

(*) Let $f$ be any complex-valued function defined on $X \cap \Delta$ such that the restrictions $f \mid M \cap \Delta$ and $f \mid N \cap \Delta$ are holomorphic. Then $f$ extends to a holomorphic function on $\Delta$.

If $X$ is quasi-normal at 0 , then $(*)$ holds for a suitable polydisk $\Delta$ (for the definition of quasi-normality, see $\S 2$ ).

It is the object of the present paper to deal with a property of varieties introduced above which implies the quasi-normality. This problem has been discussed in [2] in a restricted case. Observing examples such as Theorem 6 in [1] or Corollary 6 in [2], we are led to infer that a sense of orthogonality of $M$ to $N$ or maximality of the embedding of $X$ into $C^{d}$ at 0 has some connection with the quasinormality of $X$ and that such a situation will be well described by tangent spaces of $M$ and $N$; so we shall give a sufficient condition by use of them.

\section{§ 2. A lemma}

We denote by ${ }_{n} \mathcal{O}_{0}$ the ring of germs of holomorphic functions at 0 in $C^{n}$ and by $\mathbf{V}\left(\mathbf{f}_{1}, \cdots, \mathbf{f}_{m}\right)$ the germ of the variety defined by the ideal $\left(\mathbf{f}_{1}, \cdots, \mathbf{f}_{m}\right)$. id $\mathbf{V}\left(\mathbf{f}_{1}, \cdots, \mathbf{f}_{m}\right)$ denotes the ideal of ${ }_{n} \mathcal{O}_{0}$ consisting of germs

Received May 7, 1973. 
which vanish on the variety $\mathbf{V}\left(\mathbf{f}_{1}, \cdots, \mathbf{f}_{m}\right)$. Let $\Delta$ be a polydisk with center 0 in $C^{d}$; i.e., $\Delta=\left\{\left(z_{1}, \cdots, z_{d}\right) \in \boldsymbol{C}^{d}|| z_{i} \mid<r, i=1, \cdots, d\right\}$ for some $r>0$. Then $\Delta_{k}$ will stand for the polydisk of the same radius in $C^{k}, 1 \leqq k<d$. The ring of germs of holomorphic functions of an analytic space $X$ at a point $p \in X$ will be denoted by ${ }_{X} \mathcal{O}_{p}$, and the ring of germs of continuous, weakly holomorphic functions will be denoted by ${ }_{x} \mathcal{O}_{p}^{\prime} . X$ is said to be quasi-normal at $p$ if ${ }_{x} \mathcal{O}_{p}^{\prime}={ }_{x} \mathcal{O}_{p}$ ([1]).

The following is a generalization of Theorem 2 in [2].

LEMMA. Let $M, N$ be complex submanifolds of $\boldsymbol{C}^{d}$ of dimension $m$, $n$, respectively, and let $0 \in M \cap N$. Suppose that there exist a polydisk $\Delta$ and a nonsingular holomorphic map $\alpha=\left(f_{1}, \cdots, f_{d}\right): \Delta_{n} \rightarrow C^{d}, \alpha(0)=0$, such that

$$
M=\Delta_{m} \times\{0\} \subset C^{d}, \quad N \cap U=\alpha\left(\Delta_{n}\right)
$$

for a neighborhood $U$ of 0 in $C^{d}$. Let $X=M \cup N$. Then, $X$ is quasinormal at 0 if and only if

$$
\text { id } \mathbf{V}\left(\mathbf{f}_{m+1}, \cdots, \mathbf{f}_{d}\right)=\left(\mathbf{f}_{m+1}, \cdots, \mathbf{f}_{d}\right) .
$$

Proof. Since $\alpha$ is nonsingular, there exists a nonsingular holomorphic map $\tilde{\alpha}: \Delta \rightarrow C^{d}$ such that $\tilde{\alpha} \mid \Delta_{n} \times\{0\}=\alpha$. We may suppose that $\tilde{\alpha}$ is a biholomorphic map of $\Delta$ onto $U$.

Suppose that $X$ is quasi-normal at 0 and let $\mathbf{g} \in \mathrm{id} \mathbf{V}\left(\mathbf{f}_{m+1}, \cdots, \mathbf{f}_{d}\right)$. There exists a polydisk $\Delta^{\prime} \subset \Delta$ such that the following hold:

(1) $\tilde{\alpha}\left(\Delta^{\prime}\right) \subset U \cap \Delta$.

(2) There is a holomorphic function $g$ on $\Delta_{n}^{\prime}$ inducing the given germ $\mathbf{g}$; and $g=0$ on the subvariety $V=\left\{z^{\prime} \in \Delta_{n}^{\prime} \mid f_{j}\left(z^{\prime}\right)=0, j=m+1, \cdots, d\right\}$. Let $U^{\prime}=\tilde{\alpha}\left(\Delta^{\prime}\right)$. Then,

$$
\alpha^{-1}\left(N \cap U^{\prime}\right)=\Delta_{n}^{\prime}, \quad \alpha^{-1}\left(M \cap N \cap U^{\prime}\right)=V .
$$

We define a function $G$ on $X \cap U^{\prime}$ by

$$
G(z)= \begin{cases}0, & \text { for } z \in M \cap U^{\prime} \\ g \circ \alpha^{-1}(z), & \text { for } z \in N \cap U^{\prime} .\end{cases}
$$

$G$ is continuous and weakly holomorphic; hence there exist a polydisk $\Delta^{\prime \prime} \subset U^{\prime} \cap \Delta^{\prime}$ and a holomorphic function $\tilde{G}$ on $\Delta^{\prime \prime}$ such that $\tilde{G}=G$ on $X \cap \Delta^{\prime \prime}$. The power series expansion of $\tilde{G}$ is expressed in the form 


$$
\tilde{G}(z)=g_{0}\left(z_{1}, \cdots, z_{m}\right)+\sum_{j=1}^{r} g_{j}\left(z_{1}, \cdots, z_{m}, z_{m+j}, \cdots, z_{d}\right) z_{m+j},
$$

$z \in \Delta^{\prime \prime}$, where $r=d-m$. Let $\alpha\left(\Delta_{n}^{\prime \prime \prime}\right) \subset \Delta^{\prime \prime}$ for a suitable polydisk $\Delta^{\prime \prime \prime} \subset \Delta^{\prime \prime}$. In the above, we see that $g_{0}=0$ on $\Delta_{m}^{\prime \prime \prime}$. Putting $z=\alpha\left(z^{\prime}\right), z^{\prime} \in \Delta_{n}^{\prime \prime \prime}$, we obtain $\tilde{G}(z)=g\left(z^{\prime}\right)$; hence we have

$$
\begin{aligned}
g\left(z^{\prime}\right) & =\sum_{j=1}^{r} g_{j}\left(f_{1}\left(z^{\prime}\right), \cdots, f_{m}\left(z^{\prime}\right), f_{m+j}\left(z^{\prime}\right), \cdots, f_{d}\left(z^{\prime}\right)\right) f_{m+j}\left(z^{\prime}\right) \\
& =\sum_{j=1}^{r} a_{j}\left(z^{\prime}\right) f_{m+j}\left(z^{\prime}\right)
\end{aligned}
$$

where $a_{j}, j=1, \cdots, r$, are holomorphic functions on $\Delta_{n}^{\prime \prime \prime}$. It follows that $\mathbf{g} \in\left(\mathbf{f}_{m+1}, \cdots, \mathbf{f}_{d}\right)$.

To prove the converse, suppose that id $\mathbf{V}\left(\mathbf{f}_{m+1}, \cdots, \mathbf{f}_{d}\right)=\left(\mathbf{f}_{m+1}, \cdots, \mathbf{f}_{d}\right)$ and let $\mathbf{G} \in{ }_{X} \mathcal{O}_{0}^{\prime}$. There exists a polydisk $\Delta^{\prime} \subset U \cap \Delta$ such that the germ $\mathbf{G}$ is induced from a continuous function $G$ defined and weakly holomorphic on $X \cap \Delta^{\prime}$. We choose a polydisk $\Delta^{\prime \prime} \subset \Delta^{\prime}$ such that $\tilde{\alpha}\left(\Delta^{\prime \prime}\right) \subset \Delta^{\prime}$. Let $\pi$ be the projection: $\boldsymbol{C}^{d} \rightarrow \boldsymbol{C}^{m} \times\{0\} \subset \boldsymbol{C}^{d}$ and define a holomorphic function $G \circ \alpha-G \circ \pi \circ \alpha$ on $\Delta_{n}^{\prime \prime}$. Since $\alpha=\pi \circ \alpha$ on the subvariety

$$
V=\left\{z^{\prime} \in \Delta_{n}^{\prime \prime} \mid f_{j}\left(z^{\prime}\right)=0, j=m+1, \cdots, d\right\},
$$

it follows from the assumption that

$$
\mathbf{G} \circ \alpha-\mathbf{G} \circ \pi \circ \alpha \in\left(\mathbf{f}_{m+1}, \cdots, \mathbf{f}_{d}\right) .
$$

There exist a polydisk $\Delta^{\prime \prime \prime} \subset \Delta^{\prime \prime} \cap \tilde{\alpha}\left(\Delta^{\prime \prime}\right)$ and holomorphic functions $a_{j}$, $j=1, \cdots, r$, on $\Delta_{n}^{\prime \prime \prime}$ such that

$$
G \circ \alpha\left(z^{\prime}\right)=G \circ \pi \circ \alpha\left(z^{\prime}\right)+\sum_{j=1}^{r} a_{j}\left(z^{\prime}\right) f_{m+j}\left(z^{\prime}\right), \quad z^{\prime} \in \Delta_{n}^{\prime \prime \prime} .
$$

Let $\pi^{\prime}$ be the projection: $\boldsymbol{C}^{d} \rightarrow \boldsymbol{C}^{n}$. We define a holomorphic function; $\tilde{G}$ on a polydisk $\Delta^{\prime \prime \prime \prime}, \Delta^{\prime \prime \prime \prime} \subset \tilde{\alpha}\left(\Delta^{\prime \prime \prime}\right)$, by

$$
\tilde{G}(z)=G \circ \pi(z)+\sum_{j=1}^{r}\left(a_{j} \circ \pi^{\prime} \circ \tilde{\alpha}^{-1}\right)(z) z_{m+j}, \quad z \in \Delta^{\prime \prime \prime \prime} .
$$

$\tilde{G}$ is an extension of $G \mid X \cap \Delta^{\prime \prime \prime \prime}$. In fact, for $z \in \Delta_{m}^{\prime \prime \prime \prime} \times\{0\}$, we have $\tilde{G}(z)=G(z)$; on the other hand, if $z \in N \cap \Delta^{\prime \prime \prime \prime}$, then $z=\alpha\left(z^{\prime}\right)$ for some $z^{\prime} \in \Delta_{n}^{\prime \prime \prime}$, hence we have

$$
\tilde{G}(z)=G \circ \pi \circ \alpha\left(z^{\prime}\right)+\sum_{j=1}^{r} a_{j}\left(z^{\prime}\right) f_{m+j}\left(z^{\prime}\right)=G(z) .
$$

This completes the proof. 


\section{§ 3. Theorems}

For a complex submanifold $M$ of $C^{d}$ with $0 \in M$, the tangent space $T_{0}(M)$ to $M$ at 0 is defined to be the collection of all derivations of the ring ${ }_{M} \mathcal{O}_{0}$. We shall regard $T_{0}(M)$ as a vector subspace of $C^{d}$. Let $\operatorname{dim}_{0} M=m, \operatorname{dim}_{0} N=n$. Let $T_{0}(M, N)$ denote $T_{0}(M)+T_{0}(N)$, the subspace spanned by $T_{0}(M)$ and $T_{0}(N)$. Then, $\operatorname{dim} T_{0}(M, N)=m+n$ if and only if $T_{0}(M) \cap T_{0}(N)=(0)$; and $m+n \leqq d$ in this case. Also we have $\operatorname{dim} T_{0}(M, N)=d$ if and only if $T_{0}(M)+T_{0}(N)=C^{d}$; and $d \leqq m+n$ in this case. This means that the embedding map $\alpha: \Delta_{n} \rightarrow C^{d}$ is transversal to $M$ at 0 .

THEOREM 1. Let $M, N$ be complex submanifolds of $C^{d}$ such that $0 \in M \cap N$ and $\operatorname{dim}_{0} M=m, \operatorname{dim}_{0} N=n$. Let $X=M \cup N$. If $\operatorname{dim} T_{0}(M, N)$ $=\min (m+n, d)$, then $X$ is quasi-normal at 0 .

Proof. $M$ and $N$ are locally represented as follows in general. There exist neighborhoods $U, U^{\prime}$ of 0 in $C^{d}$, a polydisk $\Delta$, nonsingular holomorphic maps $\alpha=\left(f_{1}, \cdots, f_{d}\right): \Delta_{m} \rightarrow C^{d}, \alpha(0)=0$, and $\beta=\left(g_{1}, \cdots, g_{d}\right): \Delta_{n} \rightarrow$ $C^{d}, \beta(0)=0$, such that

$$
M \cap U=\alpha\left(\Delta_{m}\right), \quad N \cap U^{\prime}=\beta\left(\Delta_{n}\right) .
$$

Let $J_{\alpha}(0)$ and $J_{\beta}(0)$ denote the Jacobian matrices at 0 of $\alpha$ and $\beta$, respectively. The column vectors of the matrix $J_{\alpha}(0)$ constitute a basis of the tangent space $T_{0}(M)$; the situation is the same for $J_{\beta}(0)$ and $T_{0}(N)$. Hence, $\operatorname{dim} T_{0}(M, N)$ is equal to the rank of the matrix consisting of the columns of both $J_{\alpha}(0)$ and $J_{\beta}(0)$. From this follows that $\operatorname{dim} T_{0}(M, N)$ is invariant under any nonsingular change of local coordinates at 0 in $C^{d}$.

Now, we shall see that there exist complex submanifolds $M^{\prime}, N^{\prime}$ of $\boldsymbol{C}^{d}$, a neighborhood $U^{\prime \prime}$ of 0 in $\boldsymbol{C}^{d}$, a polydisk $\Delta^{\prime \prime}$ and a nonsingular holomorphic map $\gamma: \Delta_{n}^{\prime \prime} \rightarrow C^{d}$ with $\gamma(0)=0$ such that

$$
M^{\prime}=\Delta_{m}^{\prime \prime} \times\{0\} \subset C^{d}, \quad N^{\prime} \cap U^{\prime \prime}=\gamma\left(\Delta_{n}^{\prime \prime}\right),
$$

and that we can find neighborhoods $W, W^{\prime}$ of 0 and biholomorphic map $\lambda$ of $W$ onto $W^{\prime}$ for which we have $\lambda(X \cap W)=X^{\prime} \cap W^{\prime}$ where $X^{\prime}=$ $M^{\prime} \cup N^{\prime}$. In fact, let $\tilde{\alpha}, \tilde{\beta}$ be nonsingular holomorphic maps: $\Delta \rightarrow C^{d}$ such that

$$
\tilde{\alpha}\left|\Delta_{m} \times\{0\}=\alpha, \quad \tilde{\beta}\right| \Delta_{n} \times\{0\}=\beta .
$$


We may assume that $\tilde{\alpha}, \tilde{\beta}$ are biholomorphic maps of $\Delta$ onto $U, U^{\prime}$, respectively. Let $\Delta^{\prime}$ be a polydisk such that $\tilde{\alpha}\left(\Delta^{\prime}\right) \subset U \cap U^{\prime}$; let $U_{0}=$ $\tilde{\alpha}\left(\Delta^{\prime}\right)$. . Let $N^{\prime}=\tilde{\alpha}^{-1}\left(N \cap U_{0}\right)$. We define $U^{\prime \prime}=\tilde{\alpha}^{-1} \tilde{\beta}\left(\Delta^{\prime \prime}\right)$ for a polydisk $\Delta^{\prime \prime} \subset \tilde{\beta}^{-1}\left(U_{0}\right)$. We have then $N^{\prime} \cap U^{\prime \prime}=\tilde{\alpha}^{-1} \beta\left(\Delta_{n}^{\prime \prime}\right)$. Let $M^{\prime}=\Delta_{m}^{\prime \prime} \times\{0\} \subset C^{d}$, $X^{\prime}=M^{\prime} \cup N^{\prime}$. Then, we have $\tilde{\alpha}^{-1}\left(X \cap U_{0}\right)=X^{\prime}$. Therefore, it suffices to put $W=U_{0}, W^{\prime}=\Delta^{\prime}$ and $\lambda=\tilde{\alpha}^{-1}$ on $U_{0}$.

Consequently, we have only to prove that $X^{\prime}$ is quasi-normal at 0 under the assumption that $\operatorname{dim} T_{0}\left(M^{\prime}, N^{\prime}\right)=\min (m+n, d)$. Let $\gamma=$ $\left(f_{1}, \cdots, f_{d}\right)$. We define matrices $J$ and $J^{\prime}$ by

$$
\begin{gathered}
J=\left(\frac{\partial f_{i}}{\partial z_{j}}(0)\right), \quad i=1, \cdots, m ; j=1, \cdots, n, \\
J^{\prime}=\left(\frac{\partial f_{m+i}}{\partial z_{j}}(0)\right), \quad i=1, \cdots, r ; j=1, \cdots, n,
\end{gathered}
$$

where $r=d-m$. Let $A$ be the following $d \times(m+n)$ matrix where $I$ denotes the $m \times m$ identity matrix:

$$
A=\left(\begin{array}{ll}
I & J \\
0 & J^{\prime}
\end{array}\right)
$$

The condition that $\operatorname{dim} T_{0}\left(M^{\prime}, N^{\prime}\right)=\min (m+n, d)$ is equivalent to the condition that $A$ has the maximal rank, which is easily seen to be equivalent to maximality of the rank of $J^{\prime}$; this means that the map $\left(f_{m+1}, \cdots, f_{d}\right)$ is nonsingular. Thus, as in the proof of Corollary 4 in [2], we have id $\mathbf{V}\left(\mathbf{f}_{m+1}, \cdots, \mathbf{f}_{d}\right)=\left(\mathbf{f}_{m+1}, \cdots, \mathbf{f}_{d}\right)$. This completes the proof.

Since the points where a space is quasi-normal constitute an open subset, we have the following

COROLlaRY. Let $M, N$ be submanifolds satisfying the condition of Theorem 1. Then there exists a polydisk $\Delta$ such that the proposition (*) holds.

THEOREM 2. Let $\operatorname{dim}_{0}(M \cap N)=0$. Then $X$ is quasi-normal at 0 if and only if $T_{0}(M) \cap T_{0}(N)=(0)$.

Proof. Let $X$ be quasi-normal at 0 ; let $X^{\prime}=M^{\prime} \cup N^{\prime}$ and $N^{\prime} \cap U^{\prime \prime}$ $=\gamma\left(\Delta_{n}^{\prime \prime}\right)$ where $\gamma=\left(f_{1}, \cdots, f_{d}\right)$ as in the proof of Theorem 1 . The assumption implies that $\operatorname{dim}_{0} \mathbf{V}\left(\mathbf{f}_{m+1}, \cdots, \mathbf{f}_{d}\right)=0$, so $\left(\mathbf{f}_{m+1}, \cdots, \mathbf{f}_{d}\right)$ is the maximal ideal of ${ }_{n} \mathcal{O}_{0}$ and $n \leqq d-m$. We have 


$$
\mathbf{z}_{i} \in\left(\mathbf{f}_{m+1}, \cdots, \mathbf{f}_{d}\right), \quad i=1, \cdots, n,
$$

so that rank $J^{\prime}=n$ as in the proof of Proposition 5 in [2]. The result follows from $\operatorname{dim} T_{0}(M, N)=m+n$. This completes the proof.

The converse of Theorem 1 does not hold in general as is seen from the example after Corollary 4 in [2].

The variety $X$ considered in Theorem 1 has the property that $M \cap N$ is a submanifold of a neighborhood of 0 . But, we cannot expect anything significant concerning the relation between the variety $M \cap N$ and quasi-normality of $M \cup N$.

THEOREM 3. Let $V$ be an analytic subvariety of a neighborhood of 0 in $C^{n}$, and let $0 \in V$. Then there exist submanifolds $M, N, N^{\prime}$ of $\boldsymbol{C}^{d}$, $n<d$, such that $0 \in M \cap N \cap N^{\prime}, \boldsymbol{M} \cap \boldsymbol{N}=\boldsymbol{M} \cap \boldsymbol{N}^{\prime}=\boldsymbol{V} \times\{\boldsymbol{0}\} \subset \boldsymbol{C}^{d}$; and $M \cup N$ is quasi-normal, yet $M \cup N^{\prime}$ is not quasi-normal, at 0 .

Proof. Take a neighborhood $U$ of 0 and holomorphic functions $f_{1}, \cdots, f_{r}$ on $U$ such that $V \cap U=\left\{z^{\prime} \in U \mid f_{j}\left(z^{\prime}\right)=0, j=1, \cdots, r\right\}$. First, let id $\mathbf{V}\left(\mathbf{f}_{1}, \cdots, \mathbf{f}_{r}\right) \neq\left(\mathbf{f}_{1}, \cdots, \mathbf{f}_{r}\right)$. Let id $\mathbf{V}\left(\mathbf{f}_{1}, \cdots, \mathbf{f}_{r}\right)=\left(\mathbf{g}_{1}, \cdots, \mathbf{g}_{s}\right)$ for $\mathbf{g}_{i} \in{ }_{n} \mathcal{O}_{0}$, $i=1, \cdots, s$. Then we have

$$
\mathbf{V}\left(\mathbf{g}_{1}, \cdots, \mathbf{g}_{s}\right)=\mathbf{V}\left(\mathbf{f}_{1}, \cdots, \mathbf{f}_{r}\right), \quad \operatorname{id} \mathbf{V}\left(\mathbf{g}_{1}, \cdots, \mathbf{g}_{s}\right)=\left(\mathbf{g}_{1}, \cdots, \mathbf{g}_{s}\right) .
$$

Let $\Delta_{n} \subset U$ be a suitable polydisk such that $V \cap \Delta_{n}=\left\{z^{\prime} \in \Delta_{n} \mid g_{j}\left(z^{\prime}\right)=0\right.$, $j=1, \cdots, s\}$ where $g_{j}$ are holomorphic functions on $\Delta_{n}$ which are representatives of germs $\mathbf{g}_{j}$. We define submanifolds $M, N, N^{\prime}$ as follows:

$$
\begin{aligned}
M & =\Delta_{n} \times\{0\} \subset \boldsymbol{C}^{d}, \text { where } d=n+r+s, \\
N & =\left\{\left(z^{\prime}, g_{1}\left(z^{\prime}\right), \cdots, g_{s}\left(z^{\prime}\right), 0, \cdots, 0\right) \in \boldsymbol{C}^{d} \mid z^{\prime} \in \Delta_{n}\right\}, \\
N^{\prime} & =\left\{\left(z^{\prime}, f_{1}\left(z^{\prime}\right), \cdots, f_{r}\left(z^{\prime}\right), 0, \cdots, 0\right) \in \boldsymbol{C}^{d} \mid z^{\prime} \in \Delta_{n}\right\} .
\end{aligned}
$$

We have $M \cap N=M \cap N^{\prime}=\left(V \cap \Delta_{n}\right) \times\{0\} \subset C^{d} ; M \cup N$ is quasi-normal but $M \cup N^{\prime}$ is not quasi-normal, at 0 .

Next, let id $\mathbf{V}\left(\mathbf{f}_{1}, \cdots, \mathbf{f}_{r}\right)=\left(\mathbf{f}_{1}, \cdots, \mathbf{f}_{r}\right)$. It suffices to find germs $\mathbf{f}_{1}^{\prime}, \cdots$, $\mathbf{f}_{t}^{\prime} \in{ }_{n} \mathcal{O}_{0}$ such that $\mathbf{V}\left(\mathbf{f}_{1}^{\prime}, \cdots, \mathbf{f}_{t}^{\prime}\right)=\mathbf{V}\left(\mathbf{f}_{1}, \cdots, \mathbf{f}_{r}\right)$, id $\mathbf{V}\left(\mathbf{f}_{1}^{\prime}, \cdots, \mathbf{f}_{t}^{\prime}\right) \neq\left(\mathbf{f}_{1}^{\prime}, \cdots, \mathbf{f}_{t}^{\prime}\right)$. If $\left(\mathbf{f}_{1}, \cdots, \mathbf{f}_{r}\right)=(\mathbf{f}), \mathbf{f} \neq \mathbf{0}$, we have only to take $\mathbf{f}^{2}$. If $\left(\mathbf{f}_{1}, \cdots, \mathbf{f}_{r}\right)$ is not a principal ideal, we may assume that there is an integer $t, 2 \leqq t \leqq r$, such that

$$
\mathbf{V}\left(\mathbf{f}_{1}, \cdots, \mathbf{f}_{t}\right)=\mathbf{V}\left(\mathbf{f}_{1}, \cdots, \mathbf{f}_{r}\right), \quad \mathbf{f}_{1} \notin\left(\mathbf{f}_{2}, \cdots, \mathbf{f}_{t}\right) .
$$

It follows that 
$\mathbf{V}\left(\mathbf{f}_{1}^{2}, \mathbf{f}_{2}, \cdots, \mathbf{f}_{t}\right)=\mathbf{V}\left(\mathbf{f}_{1}, \mathbf{f}_{2}, \cdots, \mathbf{f}_{r}\right), \quad \operatorname{id} \mathbf{V}\left(\mathbf{f}_{1}^{2}, \mathbf{f}_{2}, \cdots, \mathbf{f}_{t}\right) \neq\left(\mathbf{f}_{1}^{2}, \mathbf{f}_{2}, \cdots, \mathbf{f}_{t}\right)$, since $\mathbf{f}_{1} \notin\left(\mathbf{f}_{1}^{2}, \mathbf{f}_{2}, \cdots, \mathbf{f}_{t}\right)$. The proof is completed.

\section{REFERENCES}

[1] N. Mochizuki, Quasi-normal analytic spaces, Proc. Japan Acad., 48 (1972), 181185.

[ 2 ] — , Quasi-normal analytic spaces. II, Kōdai Math. Sem. Rep., 25 (1973), 362366.

College of General Education

Tôhoku University 